\title{
Control abstracto de Constitucionalidad sobre reducción presupuestaria a la Educación
}

\section{Abstract control of Constitutionality on budget reduction for Education}

Grace Estefanía Méndez-Santacruz

uq.orlandoronquillo@uniandes.edu.ec

Universidad Regional Autónoma de Los Andes, Quevedo

Ecuador

https://orcid.org/0000-0002-3756-8666

Orlando Iván Ronquillo-Riera

uq.orlandoronquillo@uniandes.edu.ec

Universidad Regional Autónoma de Los Andes, Quevedo

Ecuador

https://orcid.org/0000-0001-6020-7255

Alba Rosa Pupo-Kairuz

uq.albapupo@uniandes.edu.ec

Universidad Regional Autónoma de los Andes, Quevedo

Ecuador.

https://orcid.org/0000-0002-1719-9250

Recibido: 15 de septiembre de 2020

Revisado: 20 de octubre de 2020

Aprobado: 04 de diciembre de 2020

Publicado: 11 de diciembre de 2020 
RESUMEN:

La investigación se planteó en realizar un análisis jurídico sobre las atribuciones constitucionales que tiene la Corte Constitucional respecto al control abstracto de constitucionalidad sobre reducción presupuestaria a la educación. Se trabajó en función del método analítico - sintético, develándose los aspectos fundamentales al control abstracto de constitucionalidad sobre reducción presupuestaria a la educación superior en el Ecuador. Los resultados se concretaron en determinar que la Corte Constitucional como órgano de regulación tiene la facultad de dictaminar si un acto administrativo o normativo es constitucional, asimismo se muestra una acción por incumplimiento en contra del recorte presupuestario decretado de forma inadecuada por el Ministerio de Finanzas que permitió la admisión de la reducción del presupuesto educativo y por ende la afectación de derechos colectivos.

Descriptores: Derechos humanos; derecho constitucional; derecho público. (Palabras tomadas del tesauro UNESCO).

\begin{abstract}
The investigation was proposed to carry out a legal analysis on the constitutional powers that the Constitutional Court has regarding the abstract control of constitutionality on budget reduction for education. We worked based on the analytical-synthetic method, revealing the fundamental aspects of the abstract control of constitutionality on budget reduction for higher education in Ecuador. The results were specified in determining that the Constitutional Court as a regulatory body has the power to determine whether an administrative or regulatory act is constitutional, as well as an action for non-compliance against the budget cut improperly decreed by the Ministry of Finance that allowed the admission of the reduction of the educational budget and therefore the affectation of collective rights.
\end{abstract}

Descriptors: Human rights; constitutional law; public law. (Words taken from the UNESCO thesaurus). 


\section{INTRODUCCIÓN}

Las atribuciones conferidas a la Corte Constitucional ecuatoriana son bastante amplias; por cuanto se le otorga la tarea fundamental de conservar la supremacía y la integridad de la constitución. Las competencias asignadas se destinan a generar una mejor funcionalidad cuando existe un balance equitativo de criterios. No obstante, los fallos de la Corte Constitucional se caracterizan por ser tránsitorias de cosa juzgada constitucional, lo cual indica de forma expresa que ningún funcionario podrá basar las actuaciones tomadas, sustentado en el contenido material de la norma declarada inexequible. Pese a lo expuesto, una de las confusiones más recurrentes es en relación a la terminología originada por la marcada tendencia de constitucionalistas ecuatorianos.

El control abstracto de constitucionalidad está destinado a velar por la armonía del ordenamiento jurídico, por tal razón su principal objetivo es: "garantizar la unidad y coherencia del ordenamiento jurídico a través de la identificación y eliminación de las incompatibilidades normativas (...) entre las normas constitucionales y las demás disposiciones que integran el sistema jurídico"

Como consecuencia de la reforma constitucional del año 2008 y la entrada en vigencia del nuevo texto, conforme se analizo a lo largo de esta investigación; la practica jurisprudencial en juzgados y tribunales se ha ido perfeccionado $y$ construyendo a la luz de las diferentes interpretaciones realizadas por la Corte Constitucional. La difícil tarea del juez se refleja al momento de aplicar la jurisprudencia en casos concretos, así lo detalla Zagrebelsky al mencionar que: "el juez constitucional puede disfrazar como decisión del poder constituyente lo que es una decisión no menos libre del propio juzgador" (Zagrebelsky, 2007, p. 93).

Considerando que la jurisprudencia es un conjunto de sentencias o resoluciones judiciales que repercuten en sentencias o relaciones posteriores, es decir genera un precedente judicial que permite tener una base de administración de justica enmarcada en el análisis y aplicación de la ley, se observa en esta investigación que esta se constituye en una interpretación judicial del ordenamiento jurídico enmarcada en las fuentes del derecho. Al establecer a la jurisprudencia como fuente normativa se enfoca en un contenido con sentido teórico, dogmático y técnico que 
expresan modelación jurídica. Es así como la educación es un derecho fundamental reconocido en la Constitución y establece que es obligación del Estado garantizar el acceso a la educación desde el nivel básico hasta el tercer nivel, existiendo por parte del estado una obligación de financiarla en todos los niveles.

Asimismo, la Educación Superior es concebida como un derecho humano si de instrumentos internacionales de derechos humanos se habla, así la constitución en su artículo 26 señala que "Toda persona tiene derecho a la educación. La educación debe ser gratuita, al menos en lo concerniente a la instrucción elemental y fundamental" (Constitución de la República del Ecuador, 2008).

En este sentido, (Mila, et al, 2020), resaltan el rol de la educación superior en Ecuador, es reglamentada desde la esencia de los derechos humanos y del buen vivir, este último precepto constitucional. Es necesario resaltar la importancia que los tratados internacionales de derechos humanos tienen para la legislación interna de cada país, pues se trata de ius cogens mismas que son de carácter general y obligatorio; siendo por ello necesariamente vinculantes para cualquier Estado (Cantú-Rivera, 2016).

Si bien en Ecuador se garantiza constitucionalmente el acceso a la educación superior y el progresivo financiamiento, en el marco de la emergencia Sanitaria decretada por el gobierno en torno al Coronavirus, la administración del presidente Lenin Moreno a través del Ministerio de Finanzas, dispuso la reducción en el presupuesto de Educación Superior por la cantidad de 98 millones de dólares, acto normativo contenido en él, el cual establecía "las Directrices presupuestarias para el segundo trimestre del ejercicio fiscal 2020" (Rosero, 2020).

Una vez anunciada la reducción presupuestaria, se presentaron medidas cautelares en contra de las decisiones adoptadas por el Ministerio de finanzas, pues a criterio de los accionantes eran inconstitucionales y atentaban lo establecido en el que se daba vialidad al Decreto de estado de excepción, pues consideraban que el recorte suspendía derechos constitucionales no contemplados en el decreto de estado de excepción. Debe resaltarse que la Corte Constitucional del Ecuador realiza control abstracto de constitucionalidad de las convocatorias de estados de excepción mediante sus dictámenes, así de esta forma los accionantes demandaron el 
incumplimiento del decreto que daba vialidad al estado de excepción por motivo de la pandemia.

En la solicitud de medidas cautelares se solicitaron a la Corte Constitucional, se abstengan de efectuar recortes presupuestarios hasta que se resuelvan las cuestiones de fondo planteadas en acciones de incumplimiento por parte de Universidades, organizaciones estudiantiles, y personas particulares. De esta forma el 4 de mayo del 2020, la Corte Constitucional acoge la solicitud de medidas contenidas en la acción de incumplimiento y en el dictamen se resolvió lo siguiente:

1. Disponer a las autoridades del Ministerio de Economía y Finanzas, así como a las demás autoridades de la función ejecutiva que corresponda, que suspendan y se abstengan de realizar modificaciones presupuestarias que puedan tener un impacto negativo en el presupuesto de las instituciones de educación superior. 2. Así mismo, se dispone detener los efectos del oficio circular Nro. MEF-VGF-2020- 0003-C, exclusivamente en lo que respecta al aspecto presupuestario de las instituciones de educación superior, hasta que la Corte adopte la decisión de fondo (Cáceres-Sánchez, 2020).

Ahora bien, se considera el origen del control judicial de constitucionalidad al "judicial review" estadounidense, que apareció en 1803 con ocasión del juicio Marbury versus Madison, en donde el juez Marshall afirmó la supremacía de la Constitución y estableció la facultad de los jueces para declarar la inconstitucionalidad de las leyes, amparándose en el argumento de que: "hay sólo dos alternativas demasiado claras para ser discutidas, o la Constitución controla a cualquier ley contraria a ella, o la Legislatura puede alterar la Constitución mediante una ley ordinaria. Entre tales alternativas no hay términos medios: o la Constitución es la ley suprema inalterable por medios ordinarios o se encuentra en el mismo nivel que las leyes...". (Ferrajoli, L. 2001, p.67)

Por su parte el sistema europeo de control, data de las primeras décadas del siglo XX, cuando en Austria en 1920, y Alemania en 1929 se crearon, bajo los postulados de Hans Kelsen, los tribunales constitucionales, mismos que, desaparecieron lamentablemente con motivo de la segunda gran guerra. Las ideas legadas de la Revolución Francesa, que radicaban la soberanía en la nación, soberanía que, tenía 
su expresión en el Parlamento hacedor de la ley, contribuyeron a la preeminencia de éste sobre la Función Judicial, así Gustavo Zagrebelsky, citando a Otto Mayer y su concepción del derecho administrativo, observa al Estado de Derecho como la supremacía de la ley sobre la administración, ley que es producto de la deliberación del Parlamento, otorgando a los jueces la competencia exclusiva de aplicar la ley a las controversias que surjan entre los ciudadanos, develándonos así, a un "juez boca de la ley", sin posibilidad de revisar lo hecho por el órgano legislativo.

Posteriormente en 1945, con el fin de la segunda guerra mundial, existió una suerte de rematerialización de los textos constitucionales, y con ello la importancia de los órganos de control, que precautelen como tarea fundamental la coherencia tanto formal, como material de las normas infra constitucionales con la Constitución, es ahí donde cobran fuerza los tribunales constitucionales de inspiración del maestro Kelsen, el cual veía en la conformación de éstos, por fuera de las funciones clásicas del Estado la posibilidad real de realizar un control concentrado que garantice la supremacía de la Constitución.

Ahora bien, para proceder con el control se facultaba a los miembros de los tribunales a poder contrastar el texto de una ley impugnada de inconstitucional con en el texto constitucional, y establecer así, la conformidad o inconformidad de aquella con la Constitución, dando origen al control abstracto de constitucionalidad. (Fernández-Segado, 1997).

En el Ecuador, por vez primera se contempla el control de constitucionalidad con ocasión de la Carta de 1851, encargando ésta tarea al Consejo de Estado, presentándose igual situación en las cartas políticas de 1869, 1897, y 1929; apareciendo el Tribunal de Garantías Constitucionales en la Constitución de 1945, de vida corta puesto que desapareció con la Carta de 1946, que encarga ésta tarea a la Corte Suprema de Justicia; por su parte los códigos políticos de 1967 y 1978 mantienen el esquema de Sala Especializada de la Corte Suprema, en esta última, aparece una suerte de control difuso puesto que se autoriza la inaplicación de una ley tenida por inconstitucional en los casos específicos que conoce como tribunal de justicia. 
En 1983 como expresa el autor (Sagüés, 2002), reaparece el Tribunal de Garantías Constitucionales, vale manifestar que en esos momentos el órgano de control constitucional tenía facultad para suspender los efectos de una norma reputada inconstitucional, siendo potestad del legislativo el declarar su constitucionalidad o no en última instancia; en 1997 se crea el Tribunal Constitucional, con plenas facultades para ejercer las tareas de control constitucional, hasta que finalmente y con la nueva Constitución, se cuenta con una Corte Constitucional como órgano máximo de control e interpretación del texto constitucional, así como, de los tratados internacionales de derechos humanos.

En este sentido, el objetivo de la investigación se basó en realizar un análisis jurídico sobre las atribuciones constitucionales que tiene la Corte Constitucional respecto al control abstracto de constitucionalidad sobre reducción presupuestaria a la educación.

\section{MÉTODO}

Se trabajó en función del método analítico - sintético, develándose los aspectos fundamentales al control abstracto de constitucionalidad sobre reducción presupuestaria a la educación superior en el Ecuador, en el lapso 2020, esencialmente a causa de medidas de emergencia por la pandemia por COVID-19, lo cual permitió, presentar una síntesis analítica del proceso de la corte constitucional como órgano garante de velar por el cumplimiento de los derechos fundamentales del colectivo ecuatoriano, siendo un trabajo de documentación jurídica, acorde a lo propuesto por (Lopera-Echavarría, et al., 2010).

Metodológicamente se trabajó en razón de un tipo de investigación descriptiva documental con diseño bibliográfico, lo cual, permitió revisar documentos relacionados con sentencias, prescripciones constitucionales, leyes, trabajos académicos de investigación, como muestra poblacional, lo cual, germinó en resultados para responder al objetivo de investigación, siendo la técnica empleada para tal fin, el análisis de contenido documental jurídico. 


\section{RESULTADOS}

La Corte Constitucional respecto de las acciones por incumplimiento planteadas se pronunció de fondo en ella hace un profundo análisis de los derechos alegados por los accionantes en torno a la inconstitucionalidad del recorte presupuestario a la educación destacándose múltiples situaciones:

Señaló que de conformidad con lo establecido en el artículo 141, de la Constitución de la República del Ecuador (2008), el cual declara la funcionabilidad de las funciones ejecutivas de la administración pública, lo que se entiende que constitucionalmente están facultados los distintos ministerios para implementar política pública en materia económica.

En la propicia (Constitución de la República del Ecuador, 2008), el Ministerio de Finanzas según lo determinado en el artículo 74 tiene a su cargo: "Formular y proponer, para la aprobación del presidente o presidenta de la República, los lineamientos de política fiscal inherentes a los ingresos, gastos y financiamiento, en procura de los objetivos establecidos para este fin", por tanto, la Corte estimó que el Viceministro de Finanzas actuó apegado a las normativas vigentes.

En torno a la reducción del presupuesto en educación el órgano constitucional estimó que no existe una reducción deliberada, sino que más bien este presupuesto se reduce por la caída en la reducción del Impuesto del valor agregado, teniendo por ello el efecto de una reducción de 98 millones de dólares para el ejercicio fiscal 2020. El fundamento legal de lo manifestado anteriormente se encuentra en su artículo 4 literal b el cual, establece entre otras que la educación superior se financia con "Las rentas establecidas en la Ley del Fondo Permanente de Desarrollo Universitario y Politécnico, FOPEDEUPO); de que se vea mermado el presupuesto" (Ley orgánica de Educación Superior, LOES, 2018).

Según lo establecido en el dictamen el Fondo Permanente de Desarrollo Universitario y Politécnico (FOPEDEUPO) recibe el 10\% del IVA y de la declaración al impuesto a la renta recibe el $11 \%$, por lo que evidentemente se produce una reducción en materia de presupuesto en la educación superior.

Finalmente, la Corte Constitucional desestima las acciones de incumplimiento planteadas contra él, al estimar que el Ministerio de Finanzas no está actuando al 
margen de la Constitución en cuanto a la reducción del presupuesto en educación superior. Incluso, respecto del fondo de la petición de inconstitucionalidad con anterioridad el señalo lo siguiente:

En ese sentido, el contenido del decreto de estado de excepción es el que determina el objeto del dictamen de constitucionalidad que emite la Corte Constitucional. Toda vez que el decreto presidencial que fue objeto del dictamen de constitucionalidad no hace mención a los pagos de la deuda, la Corte no realizó un control de constitucionalidad al respecto. A pesar de lo anterior, los accionantes pretenden que la Corte se pronuncie sobre una cuestión que no fue mencionada en el decreto de estado de excepción ni fue objeto del dictamen de constitucionalidad de la declaratoria de estado de excepción (Corte Constitutional del Ecuador (2020).

A partir de lo expresado, se reitera que la Constitución en su artículo Art. 28 establece que "La educación pública será universal y laica en todos sus niveles, y gratuita hasta el tercer nivel de educación superior inclusive"; difiere en gran medida en torno a los postulados de su predecesora, puesto que la misma establecía en su artículo 67 "La educación pública será laica en todos sus niveles; obligatoria hasta el nivel básico, y gratuita hasta el bachillerato o su equivalente".

Dentro de la presente investigación se ha evidenciado, como dentro de un estado de excepción se está violentando la Constitución al permitir tácitamente una reducción de rubros destinados a educación superior. Como bien manifiesta "Los estados de excepción son un verdadero shock dentro del estado de derecho, de lo que derivan dos situaciones: su aplicación indiscriminada y su necesidad en un estado de emergencia"

La acción propuesta por los accionantes no fue la adecuada, pues debió plantearse directamente una acción de inconstitucionalidad contra la Ley de educación Superior, en lo referente al financiamiento de la educación superior por medio del Fondo Permanente de Desarrollo Universitario y Politécnico (FOPEDEUPO), pues la norma claramente atenta contra la supremacía constitucional establecida en la Carta Magna. La constitución, en su artículo 98 señala que esta procede cuando cualquier acto normativo viola la norma constitucional expresa. 
En efecto según lo determinado en el artículo 348 de "Art. 348.- La educación pública será gratuita y el Estado la financiará de manera oportuna, regular y suficiente. La distribución de los recursos destinados a la educación se regirá por criterios de equidad social, poblacional y territorial, entre otros.

Como bien señala "El Tribunal Constitucional no se limita a controlar la constitucionalidad de las leyes de forma preventiva antes de que sean promulgados, sino que también lo hace de manera represiva, esto es, una vez que éstas pasan a formar parte del sistema jurídico legal. La actual Corte Constitucional debió por iniciativa propia declarar la inconstitucionalidad, pues está facultada para ello según lo establecido en el artículo 436 al estimar que la Corte Constitucional dentro de sus atribuciones puede "Declarar de oficio la inconstitucionalidad de normas conexas, cuando en los casos sometidos a su conocimiento concluya que una o varias de ellas son contrarias a la Constitución.

El control abstracto de constitucionalidad busca la unanimidad y la concordancia entre las normas que integran el sistema jurídico, es decir, actúa como un legislador negativo eliminando incompatibilidades normativas por el fondo y por la forma; dicho control es efectuado por la Corte Constitucional según lo determinado en el artículo 436 de la Carta Magna y artículo 74 de la constitución. Por ello la Corte debió ir más allá y asegurar vía dictamen el financiamiento permanente del presupuesto en educación superior (Basabe-Serrano, et al., 2010).

Otra de las características salientes de esta acción consiste en el valor de la sentencia que finaliza el procedimiento, en rigor, en el efecto de aquella que, de conformidad con la demanda, estima que la norma atacada no se adecua a alguna regla constitucional, de rango superior, aspecto que supone la invalidez de la norma controlada. Este efecto de la sentencia que acoge la demanda, usualmente señalado como valor erga omnes de la decisión esto es, más allá de la concepción del procedimiento judicial como proceso de partes, que sólo extiende el valor de la sentencia a los protagonistas del litigio. 


\section{CONCLUSIONES}

Desde la fundamentación teórica de la investigación se puede concluir que la Corte Constitucional del Ecuador, es el máximo órgano de control e interpretación en materia constitucional, pero una vez más ha quedado en relieve, que tener un órgano que ejerza este control no es garantía para que exista respeto irrestricto a la norma constitucional, pues han fallado contra norma expresa constitucional al consentir de una manera tácita que se reduzca el presupuesto a la educación.

En lo institucional, los poderes del Ejecutivo se incrementan, con la consiguiente merma en la capacidad de maniobra del Legislativo. Como consecuencia de ello, el desprestigio del Congreso crece y sus funciones se restringen a lo fiscalizador.

Con la metodología aplicada se demostró que la educación es un derecho humano universalizado, y ninguna norma de derecho interno puede restringir este principio, si constitucionalmente está expresado que la educación superior será financiada de manera oportuna, regular y suficiente, no es dable que se reduzca tal presupuesto en materia educativo porque la acción planteada no fue la correcta ante la Corte Constitucional. Es deber y obligación de la Corte en función del control abstracto de constitucionalidad, mantener la unidad y coherencia del ordenamiento jurídico interno con los postulados que la Constitución establece.

Desde el análisis jurídico se demuestra que los estados de excepción desde cualquier óptica constitucional, violentan atentan y restringen derechos, en la mayoría de los casos son los escenarios políticos y sociales perfectos para trasgredir normas fundamentales, pues constitucionalmente se consciente aquello, son un mal no tan necesario dentro de la administración pública.

Y así llegamos al final, a fuerza de eludir algunas cuestiones y varias problematizaciones que involucra el tema. Creo que esta acción, comprendida allí la que emerge de un conflicto entre poderes.

Si se agrega a estos factores, por un lado, la posición del TSJ en el Poder Judicial según la Constitución, que no está concebida como la cúspide de ese Poder estatal, ni desde el punto de vista administrativo -me refiero a los poderes de gobierno, administración y disciplinarios sobre tribunales inferiores-, ni desde el punto de vista jurídico-judicial-el TSJ no es, a semejanza de sus pares provinciales, un tribunal de 
casación que abarca la interpretación de la ley común (infra constitucional)-, sino que, por lo contrario, agrega a aquella competencia, básicamente, la del recurso absolutamente limitado de inconstitucionalidad cúspide, esta vez sí, del control difuso, a la manera angloamericana-, su configuración como tribunal constitucional resulta precisa e indiscutible.

En síntesis, la democracia ecuatoriana ha transitado a lo largo del período analizado de un extremo de bloqueo institucional a otro de personalismo unívoco. En dicho proceso, y a pesar de la promulgación de tres cartas constitucionales, el pluralismo político, la elaboración de políticas públicas estables y el equilibrio en la relación entre poderes del Estado fueron $-\mathrm{y}$ siguen siendo- residuales. Al final, la sedimentación de las instituciones políticas, el respeto al Imperio de la Ley y la construcción de una ciudadanía plena -en términos de derechos y obligacionesaún son cuestiones por construir. Sin ellas, los rendimientos del régimen democrático continuarán presentando los desniveles que este trabajo ha evidenciado.

\section{FINANCIAMIENTO}

No monetario.

\section{AGRADECIMIENTO}

A la Universidad Regional Autónoma de Los Andes, Quevedo; por motivar el desarrollo de la Investigación.

\section{REFERENCIAS CONSULTADAS}

Basabe-Serrano, S, Pachano, S, \& Mejía-Acosta, A. (2010). The Unfinished Democracy: Fundamental Rights, Political Institutions, and Govemment Performance in Ecuador (1979-2008). Revista de ciencia política (Santiago), 30(1), 65-85. https://dx.doi.org/10.4067/S0718-090X2010000100005 
Cáceres-Sánchez, N. (2020). El principio de aplicación directa de la constitución y el Control concreto de constitucionalidad en el Ecuador. [The principle of direct application of the constitution and the concrete control of constitutionality in Ecuador]. Universidad Técnica de Ambato. Facultad de Jurisprudencia y Ciencias Sociales, Unidad de Posgrado, Maestria en Derecho Constitucional. https://n9.cl/5e5i

Cantú-Rivera, H. (2016). ¿Hacia un tratado internacional sobre la responsabilidad de las empresas en el ámbito de los derechos humanos? Reflexiones sobre la primera sesión del grupo de trabajo intergubernamental de composición abierta. [Towards an International Treaty on Corporate Responsibility in the Field of Human Rights? Reflections on the First Session of the Open-Ended Intergovernmental Working Group]. Anuario mexicano de derecho internacional, 16, 425-460.

Constitución de la República del Ecuador (2008). Sección quinta. Educación. Artículo 26. [Fifth section. Education. Article 26]. Registro Oficial 449 de 20-oct-2008. Recuperado de https://n9.cl/xo9c

Corte Constitutional del Ecuador (2020). Dictamen No. 5-20-EE/20 Juez ponente: Hernán Salgado Pesantes. Recuperado de https://n9.cl/6j3nd

Fernández-Segado, F. (1997). Los inicios del control de la constitucionalidad en Iberoamérica: del control político al control jurisdiccional, en Revista Española de Derecho Constitucional, 17(49); 79-118.

Ferrajoli, L. (2001), Los Fundamentos de los Derechos Fundamentales. Madrid, editorial Trotta.

Ley orgánica de Educación Superior, LOES. (2018). Capítulo 2. Patrimonio y financiamiento de las instituciones de educación superior. [Chapter 2. Patrimony and financing of higher education institutions]. Registro Oficial Suplemento 298 de 12-oct.-2010. Última modificación: 02-ago.-2018. Recuperado de https://n9.cl/gotv

Lopera-Echavarría, J, \& Ramírez-Gómez, C, \& Zuluaga-Aristazábal, M, \& OrtizVanegas, J. (2010). El método analítico como método natural. Nómadas. Critical Journal of Social and Juridical Sciences, 25(1).

Mila, F, Maldonado, X, \& Yánez, K. (2020). Incidencia del reglamento nacional de nivelación y admisión en el acceso a la educación superior en Ecuador [Impact of the national leveling and admission regulation on access to higher education in Ecuador]. Información tecnológica, 31(3), 171184. https://dx.doi.org/10.4067/S0718-07642020000300171 
Rosero, M. (2020). Lenín Moreno: 'Universities have not used more than $85 \%$ of their Budget [Lenín Moreno: 'Universities have not used more than $85 \%$ of their budget]. Recuperado de https://n9.cl/wu0x4

Sagüés, N. (2002). Justicia constitucional y control de la ley en América Latina, en "La justicia constitucional en la actualidad, Luis López Guerra (coordinador)", Corporación Editora Nacional, Quito.

Zagrebelsky, G. (2007). Jueces Constitucionales en Teoría del Neoconstitucionalismo [Constitutional Judges in Theory of Neoconstitutionalism]. Ensayos escogidos, Edición de Miguel Carbonell, Editorial Trotta, Instituto de Investigaciones Jurídicas, UNAM, Madrid. 